FERMILAB-CONF-08-435-TD

\title{
Gradient Limitations in Room Temperature and Superconducting Acceleration Structures
}

\author{
N.A. Solyak \\ ${ }^{2}$ Fermi National Accelerator Laboratory, Batavia, IL 60510 USA
}

\begin{abstract}
Accelerating gradient is a key parameter of the accelerating structure in large linac facilities, like future Linear Collider. In room temperature accelerating structures the gradient is limited mostly by breakdown phenomena, caused by high surface electric fields or pulse surface heating. High power processing is a necessary procedure to clean surface and improve the gradient. In the best tested X-band structures the achieved gradient is exceed $100 \mathrm{MV} / \mathrm{m}$ in of $\sim 200 \mathrm{~ns}$ pulses for breakdown rate of $\sim 10^{-7}$. Gradient limit depends on number of factors and no one theory which can explain all sets of experimental results and predict gradient in new accelerating structure. In paper we briefly overview the recent experimental results of breakdown studies, progress in understanding of gradient limitations and scaling laws. Although superconducting rf technology has been adopted throughout the world for ILC, it has frequently been difficult to reach the predicted performance in these structures due to a number of factors: multipactoring, field emission, Q-slope, thermal breakdown. In paper we are discussing all these phenomena and the ways to increase accelerating gradient in SC cavity, which are a part of worldwide R\&D program.
\end{abstract}

Keywords: accelerator, acceleration gradient, Superconductivity.

PACS: $96.50 . \mathrm{Pw}, 29.17 .+\mathrm{w}$, 07.57.Hm, 84.40.-x.

\section{INTRODUCTION}

Few proposed linear collider projects in late $70^{\text {th }}-$ middle $80^{\text {th }}$ based on room temperature accelerating structures require gradient of about $100 \mathrm{MV} / \mathrm{m}$ or even higher. It was believed that gradient can be achieved in copper structures in relatively short pulses of $\sim 100 \div 400 \mathrm{~ns}$. This hope was based on some experimental data, obtained in short structures or single cavities [1]. No systematic studies of breakdown phenomena were available at that time. The first "surprise" was when few nominal NLC/JLC $1.8 \mathrm{~m}$ long structures, designed for $70 \mathrm{MV} / \mathrm{m}$ accelerating gradient were heavily damaged during processing at $50 \mathrm{MV} / \mathrm{m}$ (250ns) [2]. At the CLIC Test Facility, $30 \mathrm{GHz}$ copper structures design for $150 \mathrm{MV} / \mathrm{m}$ were processed up to of 70 $\mathrm{MV} / \mathrm{m}$ (16 ns, surface fields $304 \mathrm{MV} / \mathrm{m}$ ) with damage in the coupler iris [3]. That was a strong motivation to start systematic studies of breakdown phenomena to understand effect of different parameters on the gradient and life-time of the structure. The most important parameters can be grouped in a following categories: 1) design parameters material, type of structure (standing wave or traveling wave), group velocity, surface electric and magnetic fields, 2) RF parameters: RF power, pulse length, stored energy, pulsed temperature rise at the surface and 3) power processing parameters: processing history, breakdown rate. The effect of RF and structure parameters on gradient limit was investigated in specially designed structures, cavities and waveguides. As a result 
of studies NLC and CLIC X-band accelerating structures demonstrated accelerating gradient above $100 \mathrm{MV} / \mathrm{m}$ at $240 \mathrm{~ns}$ pulses [4-6]. Good progress was also achieved in understanding of mechanism of breakdown phenomena. Nevertheless the theory which can explain all experimental data is not built yet. Few puzzles still have no explanation in frame of existing models [6].

Gradient limitation in superconducting niobium cavities not follows the experimental scaling law for room temperature cooper structures. The best achieved gradient was $\sim 60 \mathrm{MV} / \mathrm{m}$ in very long pulses (few second, surface fields $\sim 125 \mathrm{MV} / \mathrm{m}$ ), which is much higher than prediction from scaling law for copper. It clearly shows that physics of breakdown in SC cavities are different. SC technology is very sensitive to surface defects and dirtying, it has frequently been difficult to reach the predicted performance in these structures due to a number of factors. A large number of mechanisms are involved, and the performance of any structure will be limited by whatever mechanism produces the lowest gradient limit applicable to the specific operational conditions. Quench fields, when the peak surface magnetic fields reach $200 \mathrm{mT}$, have been thought to be an ultimate limit. The recent development of the idea of layered superconductors, by Gurevich, has introduced the possibility that the $0.2 \mathrm{~T}$ field limit may not, in fact, determine an arbitrary limiting gradient in a cavity, if it is possible to use layers of insulators and high field superconductors to shield the primary current carrying element from the quench fields. In paper the field limiting mechanisms in SC cavities are discussed, as well as how these mechanisms operate, how they depend on accelerating field and what techniques might be required to mitigate them.

\section{GRADIENT LIMITATIONS IN ROOM TEMPERATURE ACCELERATING STRUCTURES}

High power processing is a necessary procedure in room temperature structure. It burns out the sharp edges and macro-particle, clean surface and improve gradient after the number of breakdown events. Typically it takes $\sim$ few hundred hours (or $>1000$ breakdowns) to reach gradient, which for the given pulse length depends on specified breakdown rate. The lower rate the lower gradient can be achieved. Experimental data for few different NLC-type X-band structures, tested in short pulses are presented in Figure 1. Breakdown rate (BDR), gradient $\boldsymbol{E}_{\boldsymbol{a}}$ and pulse length $\boldsymbol{t}_{\boldsymbol{p}}$ for each structure can be fitted by the following law [4]:

$$
\frac{E_{a}^{30} \cdot t_{p}^{5}}{B D R}=\text { const } \text {. }
$$

It was few attempts to explain physics of breakdown and breakdown probability law. Emission sites (tips of $\sim$ nm sizes) are continuously forming in strong surface electric fields $\sim 200 \mathrm{MV} / \mathrm{m}$ due to random movement of atoms at the material surface, which can explain observed Fowler-Nordheim field enhancement factors, often in the range of 30 to 100 . Two possible explanations how the emission site heating evolves to an electron cascade have been made. The first is that the tensile force on the emission tip from the applied electric field combined with the thermal stresses induced by the resistive heating result in fatigue and fracture with subsequent ionization of the 
clump of material by emitted electrons. The second is that the heating causes evaporation of molecules from the tip which has a statistical probability of ionization from the emitted current which is in turn dependent on the applied external field. Ions are then back bombarded on the surface.

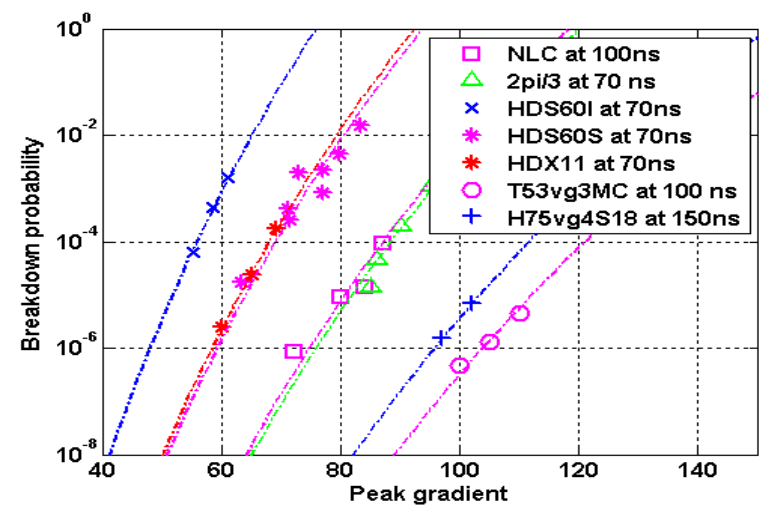

FIGURE 1: Breakdown rate vs. accelerating gradient for a few NLC-like accelerating structures, tested at short pulses after preliminary rf power processing

Energy for heating and breakdown damages comes from rf power flow in structure, which is given by the Poynting vector $\boldsymbol{S}$. Both, real and imaginary parts of power flow are relevant for tip heating. The real part of the vector $S$ is the time averaged power flow through the structure, while the imaginary part is power flow back and forth during a cycle between predominantly electric and magnetic field regions. Taking into account phase difference gives a difference in the effective coupling of real and imaginary power to the emission site. The resulting high-power limit is given by the maximum value of $S_{C}=\operatorname{Re}\{S\}+0.2 \cdot \operatorname{Im}\{S\}$ on the surface of the structure This constrain describes pretty well both TW and SW accelerating structure experimental results and is now being implemented as a design parameter for CLIC structure [4].

Breakdown studies are being done by a strong national and international collaboration. The preliminary results can be summarized as follows:

- Gradient limit have weak frequency dependence in range of 10-30 GHz.

- With the understanding of geometrical effects it was demonstrated that standing and traveling wave accelerator structures can work above 100 $\mathrm{MV} / \mathrm{m}$ loaded gradient.

- Material plays role in gradient limitations. Further understanding of materials properties may allow even greater improvements

- Experiments and recent theoretical work indicates that fatigue and pulsed heating might be the root cause of the breakdown phenomenon. Pulse heating in magnetic field in some cases is dominated in gradient limitation.

- Surface preparation is not define ultimate performances, but important for duration of power processing.

- Required gradient still have not demonstrated for full featured accelerator structure including wake field damping.

- The experimental program to date has paved the ground work for the theoretical developments. Probably few mechanisms have to be involved in 
models to explain all sets of experimental data, including different types of surface degradations as shown in Fig.2.

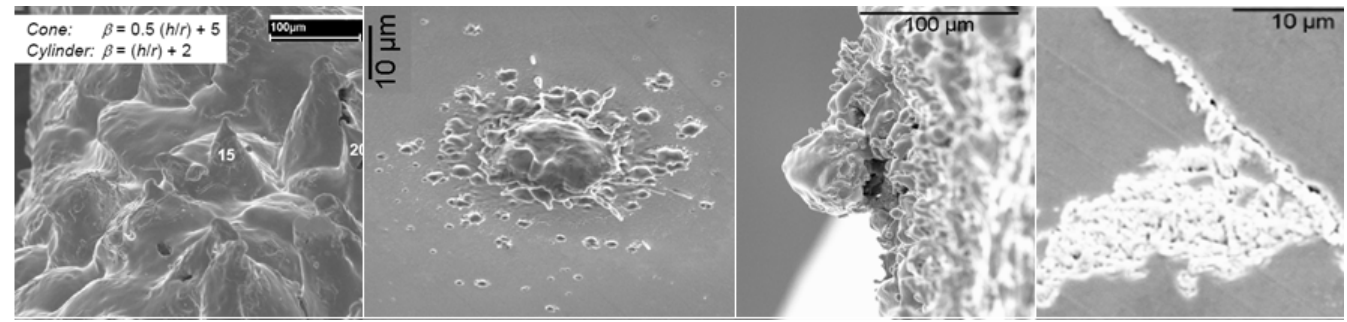

FIGURE 2. Few typical pictures of the surface degradation after breakdowns.

\section{GRADIENT LIMITATION IN SURERCONDUCTING CAVITIES}

Among big family of superconducting materials, the niobium is still the best material for RF applications. Today a hundreds of cavities are built and used in accelerator facilities around the word. Future electron-positron linear collider and large scale facilities are demanding for high gradient cavities, which bring a new breath in development of SRC physics and technology of SRF. The maximum accelerating gradient achieved in SC cavities at $1.3 \mathrm{GHz}$ is around $60 \mathrm{MV} / \mathrm{m}$ (surface field $\sim 125 \mathrm{MV} / \mathrm{m}$ ). In contrast with normal conducting structures, the pulse length is very long or $\mathrm{CW}$. It means that scaling law for copper structures not applicable for SC cavity. The typical limitation in SC cavity is not breakdown, caused by electric fields but quench or thermal breakdown, caused by superheating, mostly in area with the maximum magnetic field. What the limit for surface electric field in SC cavities is not well understood yet, but some cavities show electric field of $\sim 200 \mathrm{MV} / \mathrm{m}$. We can expect that physics of breakdown in $\mathrm{SC}$ is different from those in NC structures.

The fundamental limitation of gradient has the quantum nature. Cavity always quenches when the surface magnetic field reach critical value $\left(\mathrm{H}_{\mathrm{cr}} \approx 200 \mathrm{mT}\right.$ for $\left.\mathrm{Nb}\right)$. No one cavity exceeds this limit. But in practice the accelerating gradient in SC cavity is often limited by other factors (see Fig.3), most important of them are the following:

- Quench magnetic field: Bmax 200 mT - hard limit

- Field emission, X-ray, starts at $\sim 20 \mathrm{MV} / \mathrm{m}$, soft limit

- Thermal breakdown (strong limits for $\mathrm{f}>2 \mathrm{GHz}$ )

- Multipactor (in cavity, beampipes or couplers)

- Q-slope, increase nonlinearly with field (cryogenic losses)

- Lorentz detuning and microphonics (frequency change)

- Particulates (contaminants) - Quality of surface treatment and assembly

Surface preparation (chemistry and, especially, high pressure water rinsing) and clean assembly are essential for superconducting technology. Surface or niobium bulk contaminations are the source of field emission, multipactoring and hot spots in properly designed cavity and frequently problem disappears after repeated cycle of cavity preparation. Today big efforts are being spending on developing cavity preparation procedure, which able to provide required level of reproducibility. This is 
key issue for large scale facilities employing large number of superconducting cavities.



FIGURE 3. Typical behaviour of quality factor in ideal SC cavity (solid line - limitation by critical magnetic field) and in real cavity, where other factors limit accelerating gradient at lower level.

\section{Field emission}

Field emission (FE) produces emission current and radiation, which is a problem in both superconducting, and normal cavities and has been studied in both. In case of SC effect of beam loading due to FE is more severe, because of high quality factor. To explain typically measured dependences of the field emission current or radiation as a function of electric field, one should assume field enhancement parameters in order of $30 \div 100$. In TESLA cavity FE activity typically starts from gradient of $\sim 20 \mathrm{MV} / \mathrm{m}$. The sources of FE are particulates on the surface, which can be removed by additional high pressure water rinsing of the cavity. In some cases it can be treated by high power rf processing.

\section{Multipactor (MP)}

Multipactor is the resonant amplification of parasitic electron beams, within a cavity, caused by production of secondary electrons when these beams hit a surface. The existence of resonant trajectories can lead to electron avalanche under certain field levels and surface conditions, and can limit the performance of high power superconducting radio-frequency devices. MP can exist in surfaces with low electric field, typically at the equator of the cell, in $\mathrm{HOM}$ /fundamental couplers or in tapered beampipes. All SC cavities are designed with elliptical shape of the cell to escape or reduce MP. Though simulations show existence of resonant trajectories near equator in many elliptical cavities (for example inn TESLA cavity this is at gradient of $\sim 24$ $\mathrm{MV} / \mathrm{m}$ ) the impact energy is usually $\sim 50 \div 80 \mathrm{eV}$, small enough to have secondary emission yield is grater than 1 for clean niobium surface.

HOM coupler is another critical place, where often multipactoring conditions can be easily fulfilled: low electric fields and complicated geometry. Figure 4 shows an example of MP in HOM coupler of $3.9 \mathrm{GHz}$ cavity [7]. Aggressive high power processing caused damaging of both couplers. Problem was solved by redesigning 
HOM coupler. MP was also found in other cavities, for example ICHIRO $(1.3 \mathrm{GHz})$ and SNS $(0.8 \mathrm{GHz})$ cavities. Some tests of TESLA cavity also show soft MP at gradient of $\sim 30 \mathrm{MV} / \mathrm{m}$, which usually disappear after processing if surface is cleaned properly. ICHIRO cavity at KEK also was primary limited by multipactoring in beam

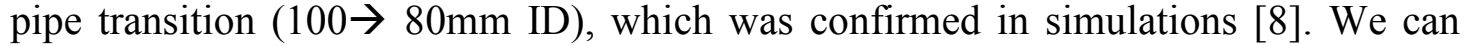
conclude that multipactor can limit accelerating gradient in SC cavity.
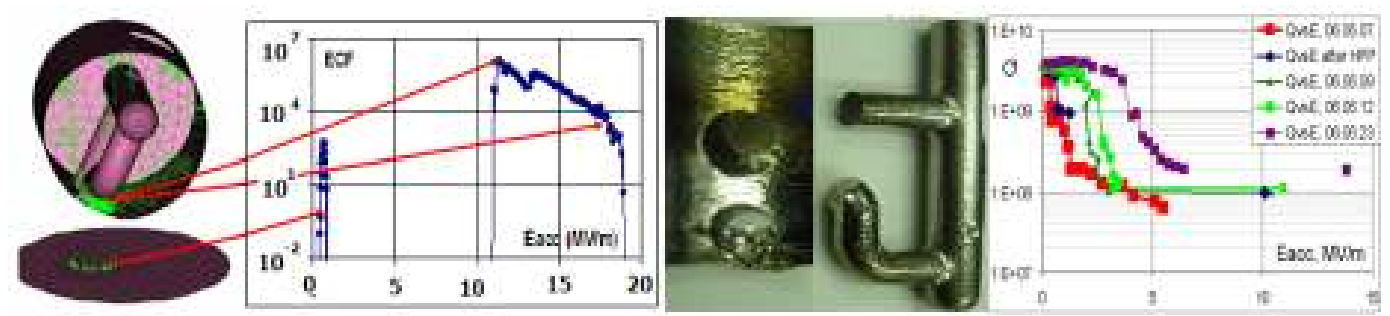

FIGURE 4. Multipactor in HOM coupler of the $3.9 \mathrm{GHz}$ cavity: Simulation shows two MP zones with different locations. Fractured HOM coupler by thermal stresses, caused by heating (middle). Test result shows Q-drop at low gradient caused by MP.

\section{Thermal breakdown}

Thermal breakdown give a very strong limitations of gradient. Often foreign defects in the bulk niobium or on the cavity surface can create hot spots, which caused thermal breakdown or quench. Local sources of hot spot can be pit or boundary between grains, which cause magnetic field enhancements (up to factor of two) and superheating. Trapped residual magnetic field create trapped magnetic fluxes, each quanta of flux is a small island of normal conducting material, which will be heated by surface current. But even ideal niobium has surface resistance and power losses. Resistance depends on temperature of surface exponentially:

$$
R_{s, B C S}^{l i n}(T)=\frac{A \cdot f^{2}}{\left(T / T_{c}\right)} e^{-\frac{\Delta}{T / T_{c}}}
$$

Where: $T$-surface temperature, $\boldsymbol{T}_{\boldsymbol{c}}=\mathbf{9 . 2 ^ { \circ }} \mathbf{K}, \boldsymbol{\Delta}=\mathbf{1 . 7 8}, f$ - frequency, $\mathrm{A}=$ constant. Power dissipated on surface increase surface temperature and results even more dissipation due to temperature dependant resistance. At some conditions this process became unstable causing thermal breakdown. Temperature will drop not only in bulk of niobium, but also in transition layer between niobium and liquid helium, known as Kapitza thermal resistance. Writing and solving equations for power transfer through surface to helium we are getting that cavity will thermally quench, when temperature rise on the surface reach his maximum value, equal:

$$
\delta T=\frac{T_{0}}{2 \cdot\left(\Delta \cdot \frac{T_{c}}{T_{0}}-1\right)}
$$

And corresponding breakdown magnetic field is equal: 


$$
H_{b}^{2}=\frac{T_{0}^{3}}{2 \cdot R_{s}\left(T_{0}\right) \cdot\left(\Delta \cdot T_{c}-T_{0}\right)} \cdot\left(\frac{k \cdot h}{k+h \cdot d}\right)
$$

Where $\boldsymbol{T}_{\boldsymbol{0}}$ - temperature of liquid helium, $\boldsymbol{k}=\boldsymbol{k}(\boldsymbol{T})$ - thermal conductivity of $\mathrm{Nb}$; $\boldsymbol{h}=\boldsymbol{h}(\boldsymbol{T})$ - Kapitza resistance. For $3.9 \mathrm{GHz}$ cavity with $2.8 \mathrm{~mm}$ wall thicknessthis formals give us: $\delta T=0.137^{\circ} \mathrm{K}$ and $\boldsymbol{H}_{b}=120 \mathrm{mT}$ for $\boldsymbol{T}_{0}=2^{\circ} \mathrm{K}$. Strong temperature dependence of surface resistance, thermal conductivity and Kapitza resistance can explain the fact that quench limit not sensitive to helium temperature at least in range $1.7-2 \mathrm{~K}$. For example, phonon peak at $2 \mathrm{~K}$ in thermal conductivity and temperature dependence of Kapitza resistance shown in Figure 5 predicts for $3.9 \mathrm{GHz}$ cavity the same quench limit in accelerating gradient $\sim 25 \mathrm{MV} / \mathrm{m}$ (magnetic field $\sim 120 \mathrm{mT}$ ) for 1.8 and $2 \mathrm{~K}$, which is in a good agreement with experimental data. This example shows that gradient in high frequency ( $\mathrm{f}>2 \mathrm{GHz}$ ) SC cavities can be limited by thermal breakdown rather than critical magnetic field [9].

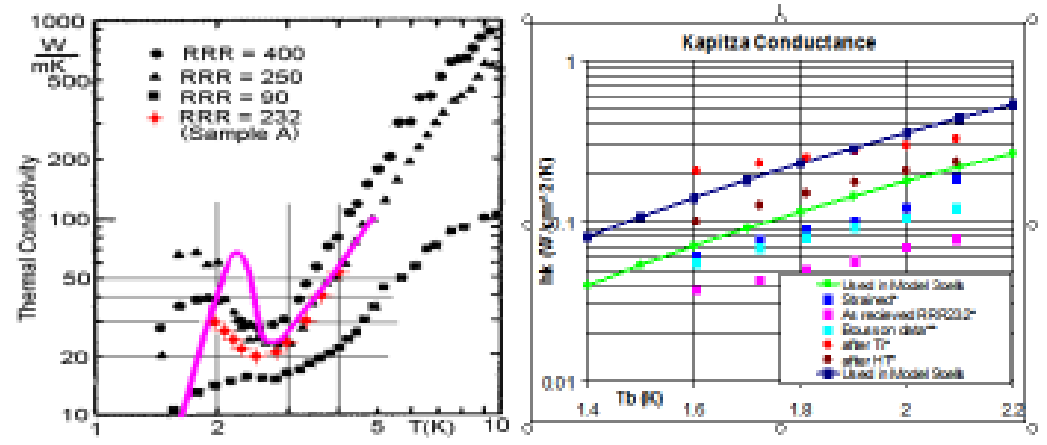

FIGURE 5. Measured thermal conductivity for niobium with different values of RRR (left) and data for Kapitza resistance (right). Data used in simulation are shown by solid lines.

\section{Push gradient limit in superconducting cavities}

Recently ideas how to increase accelerating gradient in SC cavity were proposed. First idea is the cavity shape optimization to reduce surface magnetic field and consequently increase gradient. Few designs are under investigations: low-loss (LL) cavity, reentrant (RE) cavity and traveling wave (TW) structure. In prototypes of LL cavity (KEK) and RE cavity (Cornell University) the gradient of about 50 and 60 $\mathrm{MV} / \mathrm{m}$ was successfully demonstrated [10].

Second idea is layered superconductors, proposed by A.Gurevich [11]. It was shown that the rf breakdown magnetic field of SC cavities can be significantly enhanced by multilayer coating consisting of alternating insulating layers and thin SC layers of thickness smaller than the London penetration depth. Such coating increases the vortex penetration field and the cavity quality factor determined by a smaller surface resistance in the Meissner state. Precise coating of $\mathrm{nm}$ layers is challenging task, which can be done by using Atomic Layer Deposition technique are being developed in few labs. The first results of coating are encouraging.

Power dissipation in cavity walls (and cost of cryogenic system) is growing quadratically with the increasing of accelerating gradient, while the saving from shorter length of accelerator facility is scaled linearly with the gradient. It means that 
cost optimization of whole system can limit required gradient of cavities. For ILC cost optimized accelerating gradient is somewhere around 35-40 MV/m.

\section{CONCLUSIONS}

Recent progress in reaching ultimate accelerating gradients in both normal conducting and superconducting structures became possible due to intensive experimental and theoretical R\&D efforts and international collaboration. New ideas and new materials hopefully allow push up gradients limits even higher. In each technology there are a number of other factors, which in many cases limit gradient in real cavity. Some of them can be eliminated by proper cavity design (multipactor), treatment (field emission), assembling and high power processing. Control all this factors and reproducibility of the cavity performances is the most challenging task and biggest concern for future facilities.

\section{ACKNOWLEDGMENT}

Author would like to thank I. Gonin and T.Khabiboulline for help with simulations of multipactor and thermal breakdown in SC cavity and S.Yakovlev for useful discussions and continuing interest.

\section{REFERENCES}

1. V. Balakin et al, "Accelerating structure of a Colliding Linear Electron-Positron Beam (VLEPP), Investigation of the maximum attainable acceleration rate," Novosibirsk,1978, SLAC-TRANS-0187.

2. C. Adolphsen at al, "RF Processing of X-Band Accelerator Structures at the NLCTA," LINAC2000, August, 2000, Monterey, CA.

3. W. Wuensch, "High-Gradient Breakdown In Normal-Conducting RF Cavities," EPAC 2002.

4. Grudiev, "Comprehensive Analysis of High Grandient rf Test Results and New rf Constraint" http://indico.cern.ch/conferenceTimeTable.py? confId=30911

5. V.Dolgashev, S.Tantawi, Effect of RF Parameters on breakdown Limits in High-Vacuum Xband Structures, RF 2003 workshop

6. V.Dolgashev and S.Tantawi talks on ACC08: http://aac08.lbl.gov/workinggrouptalks/WG3 Talks/Tuesday1030/

7. I.Gonin et al, Multipactor simulations in SC cavities, PAC 2007, WEPMN093

8. I.Hwang et.al, "Multipacting study of ICHIRO end cavity", RF2007 workshop

9. E.Harms, et al., "Status of 3.9 GHz SC RF cavity technology at Fermilab", RF2007 workshop

10. R.L. Geng et. al., PAC07_WEPMS006

11. Gurevich, Appl. Phys. Lett. 88, 012511 (2006) 